\title{
Neuromuscular Adaptations after Blood Flow Restriction Training Combined with Nutritional Supplementation: A Preliminary Study
}

\author{
Ivan Chulvi-Medrano ${ }^{1}$, Moises Picon-Martinez ${ }^{1}$, Miguel Garcia-Jaen ${ }^{1}$, \\ Juan Manuel Cortell-Tormo ${ }^{1}$, Yasser Alakhdar'2, Gilberto Laurentino ${ }^{3}$
}

\begin{abstract}
Affiliations: 'University of Alicante, Faculty of Education, Department of General and Specific Didactics, Alicante, Spain, ${ }^{2}$ University of Valencia. Faculty of Physiotherapy, Valencia, Spain, ${ }^{3}$ University of Sao Paulo, Department of Biodynamics of the Human Body Movement, Sao Paulo, Brasil
\end{abstract}

Correspondence: J. M. Cortell-Tormo, University of Alicante, Faculty of Education, Department of General and Specific Didactics, 03960 - San Vicente del Raspeig s/n, Alicante, Spain. E-mail: jm.cortell@ua.es

ABSTRACT Blood flow restriction training (BFRT) has been shown to be an effective alternative technique to conventional resistance training to increase skeletal muscle hypertrophy and strength. However, neuromuscular response to BFRT in combination with nutritional supplementation has rarely been studied. The purpose of this study was to investigate the effects of BFRT combined with creatine monohydrate $(\mathrm{CrM})$ and/or hydroxymethyl butyrate $(\mathrm{HMB})$ on skeletal muscle size and strength. Fifteen healthy males were randomly divided into three groups: a) BFRT without supplementation (C-BFR); b) BFRT with CrM supplementation $\left(0.3 \mathrm{~g} / \mathrm{kg}^{-1}\right)(\mathrm{CrM}-\mathrm{BFR})$; and c) BFRT with $\mathrm{CrM}\left(0.3 \mathrm{~g} / \mathrm{kg}^{-1}\right)$ and $\mathrm{HMB}(3 \mathrm{~g})$ supplementation (CrM + HMB-BFR). Participants performed elbow flexion exercise (30\% of maximal isometric voluntary contraction (MIVC)) at $30 \%$ of total occlusion pressure, twice a week, for three weeks. MIVC of the elbow flexion and brachial biceps muscle thickness were assessed pre- and post-training. There was no significant group-by-time interaction for MIVC values $(\mathrm{p}>0.05)$. Biceps muscle thickness was significantly increased from pre- to post-test in all groups $(\mathrm{p}<0.05)$. The $\mathrm{C}$-BFR group obtained a greater value of effect size $(\mathrm{d}=2.2)$. These findings suggest that 3 weeks of CrM and HMB supplementation had no influence on BFRT-induced neuromuscular adaptations.

KEY WORDS Skeletal muscle hypertrophy, muscle strength, occlusion training.

$@$ MJSSMontenegro

BLOOD FLOW RESTRICTION TRAINING

http://mjssm.me/?sekcija=article\&artid=171

\section{Introduction}

Resistance training (RT) is essential to promoting neuromuscular adaptations in a large range of the population. Consequently, the American College of Sports Medicine (ACSM) guidelines recommend using loads of $\geq 70 \%$ of one repetition maximum (1RM) to achieve muscular hypertrophy and strength gains (American College of Sports Medicine, 2009). While these guidelines are appropriate for healthy individuals, inactive adults and clinical populations may have significant limitations to using the load of RT recommended for ACSM; therefore, other options are necessary to improve musculoskeletal fitness (Chulvi-Medrano, 2011). For these populations, low-load resistance training (20\%-30\% 1RM) with blood flow restriction (LL-BFR) has been deemed an alternative technique to conventional RT (Chulvi-Medrano, 2011; Jeremy Paul Loenneke \& Pujol, 2009; Pope, Willardson, \& Schoenfeld, 2013; Slysz, Stultz, \& Burr, 2016). This training technique, also called $\mathrm{Kaatsu}^{\circ}$ training, has been shown to be an effective method for increasing muscle size and strength in clinical settings and athletic populations (Amani, Sadeghi, \& Afsharnezhad, 2018; Martín-Hernández, Marín, 2011; Martín-Hernández, 2011; Meester, Stodden, Brian, True, \& Cardon, 2016). During LL-BFR exercise, partial blood flow restriction of the exercised muscle is achieved by proximal compression of the limb. LLBFR training usually consists of 2-3 sessions per week, 3-5 sets of 15 repetitions or sets carried out to volition- 
al failure, and 30-60-second rest intervals between sets (Martín-Hernández, 2011).

Although the exact mechanisms underlying BFRT-induced neuromuscular adaptations remain unclear, some candidates have been depicted, such as intramuscular metabolite production (i.e., lactate accumulation), increases in acute secretion of growth hormone $(\mathrm{GH})$, catecholamine, and inorganic phosphate (Pi) (Loenneke, Fahs, Rossow, Abe, \& Bemben, 2012; Loenneke \& Pujol, 2009), depletion of muscle phosphocreatine (PCr) and decreased pH (Loenneke \& Pujol, 2009). Lastly, increases of skeletal muscle fast-twitch fibres recruitment and muscle cell swelling would contribute to the stimulation of different anabolic-signalling pathways (Loenneke et al., 2012).

Nutritional supplementation combined with RT has been shown to yield significant increases in muscle size, strength, and power in greater magnitude than RT alone (Buford et al., 2007; Helms, Aragon, \& Fitschen, 2014; Pearson, Hamby, Russel, \& Harris, 1999). Specifically, creatine monohydrate (CrM) and hydroxymethyl butyrate (HMB) supplementation combined with RT have been shown to be effective for inducing neuromuscular adaptations in trained and untrained individuals (Buford et al., 2007; Helms et al., 2014; Portal, Eliakim, Nemet, Halevy, \& Zadik, 2010; Wilson et al., 2013). Oral intake of CrM increases both intramuscular creatine and phosphocreatine concentrations leading to a concomitant increase in body mass and performance (Balsom, Söderlund, \& Ekblom, 1994; Birch, Noble, \& Greenhaff, 1994; Helms et al., 2014). These ergogenic effects may be caused by the availability of the amino acid leucine and some derivative metabolites, since the $\beta$-isocaproate inhibits proteolysis (Nair, Schwartz, \& Welle, 1992; Nissen et al., 1996). The positive effects of RT with HMB supplementation are also consistent. Jówko et al. (2001) demonstrated in untrained individuals that the increases skeletal muscle mass and strength following RT with HMB were superior in comparison to those obtained with RT alone after 3 weeks of training. In addition, Wilson et al. (2014) found that this strategy was effective to enhance RT training-induced gains in strength, power, and muscle mass in resistance-trained males.

While there is a growing body of evidence highlighting the potential benefits of LL-BFR exercise on skeletal muscle hypertrophy and strength, the effects of this strategy combined with nutritional supplementation on neuromuscular adaptations responses remain unknown.

Therefore, this study aimed to investigate the effects of an LL-BFR exercise programme with and without CrM and HMB supplementation on skeletal muscle hypertrophy and strength adaptations. We hypothesized that the LL-BFR-induced hypertrophy and strength increases combined with CrM and HMB would overcome those obtained with LL-BFR alone, since the CrM and HMB supplementation combined with conventional RT has shown a synergistic effect (Jówko et al., 2001).

\section{Methods and materials}

\section{Participants}

Fifteen healthy male untrained college students (age: $23.3 \pm 2.6$ years; body mass: $78.1 \pm 12.68 \mathrm{~kg}$; height 1.78 $\pm 0.07 \mathrm{~m}$ ) were recruited to participate in this study. The participants were randomly allocated to one of the three groups: a) blood flow restriction without supplementation $(C-B F R, n=5)$, b) blood flow restriction with creatine monohydrate supplementation $(\mathrm{CrM}-\mathrm{BFR}, \mathrm{n}=5)$, and $\mathrm{c}$ ) blood flow restriction with creatine monohydrate plus hydroxymethyl butyrate supplementation $(\mathrm{CrM}+\mathrm{HMB}-\mathrm{BFR}, \mathrm{n}=5)$. The criteria for inclusion in the study were: resistance-trained men who were 20-30 years old with at least 6 months of experience in RT. Participants with cardiovascular risk, food intolerances, or specific allergies to the supplements, neuromuscular disorders or any acute or chronic disease were excluded from the study. Likewise, all participants should not have used nutritional supplementation or drugs within six months prior to the commencement of the study. The study was approved by the Ethics Committee of the University of Valencia (procedure number H1419281092018) and conducted accordingly with the Declaration of Helsinki. Following the explanation about the risks and benefits of this study, participants signed their written informed consent.

\section{Procedures}

Anthropometric measurements (body mass, height, body mass index), maximal isometric voluntary contraction (MIVC), biceps muscle thickness (BMT), and total occlusion pressure (TOP) were obtained prior the study. After two familiarization sessions of testing and protocols, participants were randomly divided into three training groups: a) blood flow restriction without supplementation (C-BFR), b) blood flow restriction with creatine monohydrate supplementation (CrM-BFR), and c) blood flow restriction with creatine monohydrate plus hydroxymethyl butyrate supplementation (CrM + HMB-BFR). All participants performed the biceps curl exercise at 30\% of MIVC and 30\% of TOP, 3 sets of 15 repetitions, twice per week, for three weeks. Elbow flexion strength and biceps muscle thickness were assessed before and after training.

\section{Participant's characteristics and anthropometric measurements}

Body mass $(\mathrm{kg})$ was measured with the participants in a standing position on electrical bioimpedance equipment (Omrom - HbF-510W), and height $(\mathrm{m})$ was measured with each participant at the same position on a stadiometer (Seca $\left.{ }^{\oplus} 217\right)$.

Maximal isometric voluntary contraction (MIVC). Isometric strength was evaluated through MIVC from the dominant arm of each participant using a load cell (Mutonic ${ }^{\circ}$ SP51 "HiLine" V6.10). The participants 
were placed in a standing position, and their elbow positioned at $90^{\circ}$ of flexion and monitored with an analogic goniometer. Participants performed two attempts of maximal voluntary isometric contractions for five seconds, with rest periods of 180s between them. In short, participants were instructed to perform an elbow flexion action as forcefully as possible during contraction time. The highest value of two attempts was used for statistical analysis.

Bicep muscle thickness. Bicep muscle thickness was recorded using an ultrasonographic technique (Sonosite M-Turbo). The measurements were performed with the participants in a supine position with their arms extended and relaxed. Before measurements, the participants rested for $15 \mathrm{~min}$ to allow fluid shifts to occur and all muscle thickness measurements were performed by an experienced physiotherapist. Biceps muscle thickness values were measured by placing the probe perpendicular without depressing the skin at the specific landmark that was identified at $60 \%$ of the distance from the acromion process of the scapula to the lateral epicondyle of the humerus.

Total occlusion pressure (TOP). The value of TOP was individually registered through Doppler ultrasound. For this measure, a pressure cuff $(57 \mathrm{~cm}$ length $\times 9 \mathrm{~cm}$ width; Riester Komprimeter, Riester, Jungingen, Germany) was attached to participant's axillar region and then progressively inflated, while the arterial blood flow was monitored using Doppler ultrasound. When brachial arterial blood flow was interrupted, the arterial occlusion pressure level was recorded (100\% of TOP). For all BFR protocols, $30 \%$ of TOP was used.

Training protocols. Prior to starting the training protocols, participants performed two familiarization sessions with LL-BFR exercise at $20 \%$ of MIVC and $20 \%$ of TOP. A $48 \mathrm{~h}$ interval was allowed between familiarization sessions and testing before starting the training programme. For training protocols, all participants performed the biceps curl exercise at 30\% of MIVC and 30\% of TOP individual with the dominant arm. In each training session, the participants performed 3 sets of 15 repetitions with a 60 -second interval between sets and a contraction cycle duration of 2 seconds in the concentric phase and 2 seconds in the eccentric phase. The pressure restriction was maintained throughout the exercise and rest intervals. The training programme was carried out with a frequency of 2 days per week (Monday and Thursday), for 3 weeks. All training sessions were supervised by a personal trainer.

Nutritional supplementation. Prior to the study, participants recorded their dietary pattern for a week, and a nutritionist instructed the participants to maintain their normal diet based on the data collected in the study. Besides normal dietary, the participants from the CrM-BFR group was supplemented with creatine monohydrate (rate of $0.3 \mathrm{~g} / \mathrm{kg}$ body mass), whereas the participants from CrM + HMB-BFR group received the same amount of creatine monohydrate of the CrM-BFR group combined with $3 \mathrm{~g}$ of supplementation of hydroxymethyl butyrate (Scitec Nutrition ${ }^{\circ}$ ). The C-BFR group received no supplementation. Nutritional supplementation doses used in this study were based on the findings of previous studies (Buford et al., 2007; Wilson et al., 2013). Nutritional supplementation was administered 10 minutes before beginning each training session in a double-blind fashion.-

\section{Statistical analysis}

The results are presented as mean and standard deviations (SD). The comparison between paired samples (intragroup) was carried out using a $t$-test to compare maximal isometric voluntary contraction and biceps muscle thickness in each group before and after the training programme. Adjustment for multiple comparisons was made with Bonferroni's correction. The analysis of variance (two-factor ANOVA) was also performed followed by post hoc DMS multiple comparisons to make intergroup comparisons. The level of significance was set at $\mathrm{p}<0.05$. SPSS 18.0 software, licensed from the University of Alicante, was used for statistical analysis. The effect size was calculated using the Cohen test ([post-test mean -pre-test mean] / pre-test standard deviation). To determine the effect size of the intervention, applied the values for trained subjects were applied (Rhea, 2004): trivial effect $\mathrm{d}<0.25$; small effect $\mathrm{d}=0.25-0.50$; moderate effect $\mathrm{d}=0.50-1.0$; large effect $\mathrm{d}>1.0$. Finally, the percentage increase in each group was calculated using the following formula: (post-intervention mean - pre-intervention mean) / pre-intervention mean $\times 100$.

\section{Results}

Maximal isometric voluntary contraction

There was no time effect $x$ interaction for MIVC for all groups from pre to post-test ( $p>0.05)$. MIVC was increased in $11.20 \%, 31.60 \%$, and $3.66 \%$ in the C-BFR, CrM-BFR and CrM + HMB-BFR groups, respectively, from pre- to post-training (Table 1). No significant increase was found between groups at pre-test and posttest $(\mathrm{p}>0.05)$.

\begin{tabular}{lcccc}
$\begin{array}{l}\text { TABLE } 1 \text { Maximal Isometric Voluntary Contraction for C-BFR, CrM-BFR, and CrM + HMB-BFR Groups } \\
\text { from Pre- to Post-test }\end{array}$ & $\begin{array}{c}\text { Pre-test } \\
\text { M } \pm \text { SD (in kg) }\end{array}$ & $\begin{array}{c}\text { Post-test } \\
\text { M } \pm \text { SD (in kg) }\end{array}$ & $\boldsymbol{p}$ & Cohen $\boldsymbol{d}$ \\
\hline Groups & $17.04 \pm 2.7$ & $18.97 \pm 3.17$ & 0.444 & 0.71 \\
\hline C-BFR & $14.24 \pm 5.7$ & $18.74 \pm 4.06$ & 0.094 & 0.78 \\
CrM-BFR & $16.08 \pm 2.9$ & $16.67 \pm 3.5$ & 0.377 & 0.20 \\
CrM + HMB-BFR & & &
\end{tabular}




\section{Biceps muscle thickness}

There was a significant main effect of time and group interaction for biceps muscle thickness $(p<0.05)$. Biceps muscle thickness was increased in $12.5 \%, 8.88 \%$, and $13.12 \%$ in the C-BFR, CrM, and CrM + HMB-BFR groups respectively, from pre- to post-training (Table 2). There was a significant difference between the C-BFR group and CrM + HMB-BFR group at post-training $(\mathrm{p}<0.05)$.

\begin{tabular}{lcccc}
\multicolumn{4}{l}{ TABLE 2 Outcomes for the Biceps Muscle Thickness Before (pre-test) } & \multicolumn{4}{c}{ And After (post-test) Intervention } \\
Groups & $\begin{array}{c}\text { Pre-test } \\
\text { M } \pm \text { SD (in kg) }\end{array}$ & $\begin{array}{c}\text { Post-test } \\
\text { M } \mathbf{S D} \text { (in kg) }\end{array}$ & $\boldsymbol{p}$ & Cohen $\boldsymbol{d}$ \\
\hline C-BFR & $3.52 \pm 0.20$ & $3.96 \pm 0.15$ & $0.003^{*}+$ & 2.2 \\
CrM-BFR & $3.49 \pm 0.31$ & $3.80 \pm 0.25$ & $0.031^{*}$ & 1.0 \\
CrM + HMB-BFR & $3.20 \pm 0.33$ & $3.62 \pm 0.17$ & $0.048^{*}$ & 1.27 \\
\hline
\end{tabular}

Note: * Significant difference from pre- to post-training $(p<0.05)$; $†$ Significant difference between C-BFR group and $\mathrm{CrM}$ + HMB-BFR group at post-training $(p<0.05)$.

\section{Discussion}

The main findings of this investigation were: a) Isometric strength was not influenced by blood flow restriction training or nutritional supplementation; b) Low-load RT with blood flow restriction induced increases in biceps muscle hypertrophy regardless of nutritional supplementation.

\section{Maximal isometric strength}

Despite the fact that other studies have demonstrated an advantage in strength gains in applying low-load resistance training with blood flow restriction compared to the same protocol without BFR (Slysz et al., 2016) and a similarity to high-load resistance training (Laurentino et al., 2012; Takarada et al., 2000), in our study we did not observe changes in isometric peak torque of elbow flexors following 3 weeks of BFRT. Similar findings were reported in a recent study in which the isometric peak torque of the knee extensors was not significantly changed following 6 weeks BFRT (Cook, Scott, Hayes, \& Murphy, 2018). In addition, in this study, a reduction of $2 \%$ in the central activation was observed. These results suggest that the involvement of the central nervous system following BFR resistance exercise as strength improvements may not be of neural origin.

In contrast, in Cook's study, a significant increase of $13 \%$ in leg extension 1RM was observed. Although we did not evaluate elbow flexors 1RM in our study, it has been suggested that nonspecific strength assessment, such as in isometric of isokinetic testing, may more precisely reflect the response to different training protocols (Buckner et al., 2017). Regarding the overall strength observed in the present study, we can speculate that the training mode through of isotonic contractions and the lack of significant change in isometric muscle strength may mask neural adaptations assessed through an isometric contraction. Altogether, the low interference of LL-BFR on neural adaptation and testing specificity could have influenced the response in strength gains after the training period. However, short-term training ( 3 weeks) perhaps would have been insufficient to reach significant changes in strength following LL-BFR.

\section{Muscle hypertrophy and nutritional supplementation}

Regarding increases in muscle mass, our results showed that BFR protocols were efficient to increase muscle hypertrophy, regardless of nutritional supplementation. These data are consistent, since a variety of studies has pointed out higher muscle mass gain in LL-BFR in comparison to LL without BFR (Slysz et al., 2016) and similar effects to high-load resistance exercise (Laurentino et al., 2012; Martín-Hernández et al., 2013). However, no study to our knowledge has investigated the effect of nutritional supplementation combined with LL-BFR on muscle size. The Position Stand of the International Society of Sports Nutrition has demonstrated the ergogenic effects of supplementation of CrM combined with RT (Buford et al., 2007). For instance, Souza-Junior et al. (2011) reported increases in maximal strength, isokinetic peak torque and muscle mass after 8 weeks of RT programme (8-10 RM) in bench press and back squat exercises associated with the supplementation of $20 \mathrm{~g}$ of CrM. In another study, Antonio and Ciccone (2013) demonstrated that $5 \mathrm{~g}$ of creatine monohydrate ingestion post-exercise, twice a week, induced increase in fat-free mass and loss of fat mass following a 4-week periodized-resistance training programme ( 3 sets $\times 5$-10 reps).

Previous research has evaluated the effectiveness of CrM combined with HMB supplementation on muscle mass and strength gains, and the results are conflicting (Jówko et al., 2001; O’Connor \& Crowe, 2007). Jówko et al. (2001) reported significant increases in muscle mass and strength in a group supplemented with CrM + HMB compared to a placebo-supplemented group after three weeks of RT using a progressive load programme. In contrast, O'Connor and Crowe (O’Connor \& Crowe, 2007) demonstrated no additional effect of oral co-administration of CrM (3g) and $\mathrm{HMB}(3 \mathrm{~g})$ on muscle strength after six weeks of RT. Our results showed no additional effect of HMB and CrM plus HMB supplementation associated with LL-BFR on increases in muscle mass and maximum isometric strength. These data are in accordance with those obtained in a recent metanalysis published by Sanchez-Martinez et al. (2018), which highlighted no ergogenic effect of $\mathrm{HMB}$ on the morphological and functional musculoskeletal system in trained subjects. 
Lastly, the discrepancies between our findings compared to the previous studies regarding nutritional supplementation combined with LL-BFR may be, at least partially, explained by the differences in the dose of supplementation, the duration of the study, and the load used in LL-BFR protocols.

In conclusion, three weeks of LL-BFR induced increases in muscle hypertrophy but not on strength gains. In addition, CrM and CrM plus HMB supplementation showed no additional effect of LL-BFR-induced neuromuscular adaptations.

The current study reveals some potential limitations. First, this is a preliminary study, and the small sample size limits statistical power. Second, the lack of dietary monitoring using a normalized isocaloric diet also could have affected our results. Third, an LI-BFR with HMB supplementation and placebo groups could be included in study design. Lastly, the duration of this study could be longer (more than 4-6 weeks). -

\section{Acknowledgements}

The authors would like to thank all the participants of this study, as well as Miguel García-Jaén who participated in this study supported by a pre-doctoral grant ACIF/2016/048 from the Generalitat Valenciana, Spain.

\section{REFERENCES}

Amani, A. R., Sadeghi, H., \& Afsharnezhad, T. (2018). Interval Training with Blood Flow Restriction on Aerobic Performance among Young Soccer Players at Transition Phase. Montenegrin Journal of Sports Science and Medicine, 7(2), 5-10. doi: 10.26773/mjssm.180901

American College of Sports Medicine. (2009). Progression Models in Resistance Training for Healthy Adults. Medicine \& Science in Sports \& Exercise, 41(3), 687-708. doi: 10.1249/MSS.0b013e3181915670

Antonio, J., \& Ciccone, V. (2013). The effects of pre versus post workout supplementation of creatine monohydrate on body composition and strength. Journal of the International Society of Sports Nutrition, 10(1), 36. doi: 10.1186/1550-2783-10-36

Balsom, P. D., Söderlund, K., \& Ekblom, B. (1994). Creatine in humans with special reference to creatine supplementation. Sports Medicine (Auckland, N.Z.), 18(4), 268-280. Retrieved from http://www.ncbi.nlm. nih.gov/pubmed/7817065

Birch, R., Noble, D., \& Greenhaff, P. L. (1994). The influence of dietary creatine supplementation on performance during repeated bouts of maximal isokinetic cycling in man. European Journal of Applied Physiology and Occupational Physiology, 69(3), 268-276. Retrieved from http://www.ncbi.nlm.nih.gov/ pubmed/8001541

Buckner, S. L., Mouser, J. G., Dankel, S. J., Jessee, M. B., Mattocks, K. T., \& Loenneke, J. P. (2017). The General Adaptation Syndrome: Potential misapplications to resistance exercise. Journal of Science and Medicine in Sport, 20(11), 1015-1017. doi: 10.1016/j.jsams.2017.02.012

Buford, T. W., Kreider, R. B., Stout, J. R., Greenwood, M., Campbell, B., Spano, M., ... Antonio, J. (2007). International Society of Sports Nutrition position stand: creatine supplementation and exercise. Journal of the International Society of Sports Nutrition, 4(1), 6. doi: 10.1186/1550-2783-4-6

Chulvi-Medrano, I. (2011). Resistance training combined with superimposed partial occlusion. A review. Revista Andaluza de Medicina Del Deporte, 4(3), 121-128.

Cook, S. B., Scott, B. R., Hayes, K. L., \& Murphy, B. G. (2018). Neuromuscular Adaptations to Low-Load Blood Flow Restricted Resistance Training. Journal of Sports Science \& Medicine, 17(1), 66-73. Retrieved from http://www.ncbi.nlm.nih.gov/pubmed/29535579

Helms, E. R., Aragon, A. A., \& Fitschen, P. J. (2014). Evidence-based recommendations for natural bodybuilding contest preparation: nutrition and supplementation. Journal of the International Society of Sports Nutrition, 11(1), 20. doi: 10.1186/1550-2783-11-20

Jówko, E., Ostaszewski, P., Jank, M., Sacharuk, J., Zieniewicz, A., Wilczak, J., \& Nissen, S. (2001). Creatine and beta-hydroxy-beta-methylbutyrate (HMB) additively increase lean body mass and muscle strength during a weight-training program. Nutrition (Burbank, Los Angeles County, Calif.), 17(7-8), 558-566. Retrieved from http://www.ncbi.nlm.nih.gov/pubmed/11448573

Laurentino, G. C., Ugrinowitsch, C., Roschel, H., Aoki, M. S., Soares, A. G., Neves, M., ... Tricoli, V. (2012). Strength training with blood flow restriction diminishes myostatin gene expression. Medicine and Science in Sports and Exercise, 44(3), 406-412. doi: 10.1249/MSS.0b013e318233b4bc

Loenneke, J. P., Fahs, C. A., Rossow, L. M., Abe, T., \& Bemben, M. G. (2012). The anabolic benefits of venous blood flow restriction training may be induced by muscle cell swelling. Medical Hypotheses, 78(1), 151-154. doi: 10.1016/j.mehy.2011.10.014

Loenneke, J. P., \& Pujol, T. J. (2009). The Use of Occlusion Training to Produce Muscle Hypertrophy. Strength and Conditioning Journal, 31(3), 77-84. doi: 10.1519/SSC.0b013e3181a5a352

Martín-Hernández, J, Marín PJ, H. A. (2011). Review of muscle hypertrophy mechanisms induced by resistance occlusive training. Revista Andaluza de Medicina Del Deporte, 4(4), 129-177.

Martín-Hernández, J, B. R. H. A. (2011). Physical exercise supplemented with tisular ischemia prevents muscle atrophy. Archivos de Medicina Del Deporte, 28(145), 383-393.

Martín-Hernández, J., Marín, P. J., Menéndez, H., Ferrero, C., Loenneke, J. P., \& Herrero, A. J. (2013). Muscular adaptations after two different volumes of blood flow-restricted training. Scandinavian Journal 
of Medicine \& Science in Sports, 23(2), e114-20. doi: 10.1111/sms.12036

Meester, A. De, Stodden, D., Brian, A., True, L., \& Cardon, G. (2016). Associations among Elementary School Children's Actual Motor Competence, Perceived Motor Competence, Physical Activity and BMI : A Cross-Sectional Study Associations among Elementary School Children's Actual Motor Competence, Perceived Motor Compet, (October), 1-14. doi: 10.5061/dryad.96cp2

Nair, K. S., Schwartz, R. G., \& Welle, S. (1992). Leucine as a regulator of whole body and skeletal muscle protein metabolism in humans. American Journal of Physiology-Endocrinology and Metabolism, 263(5), E928-E934. doi: 10.1152/ajpendo.1992.263.5.E928

Nissen, S., Sharp, R., Ray, M., Rathmacher, J. A., Rice, D., Fuller, J. C., ... Abumrad, N. (1996). Effect of leucine metabolite beta-hydroxy-beta-methylbutyrate on muscle metabolism during resistance-exercise training. Journal of Applied Physiology (Bethesda, Md. : 1985), 81(5), 2095-2104. doi: 10.1152/jappl.1996.81.5.2095

O'Connor, D. M., \& Crowe, M. J. (2007). Effects of Six Weeks of $\beta$-Hydroxy- $\beta$-Methylbutyrate (HMB) and $\mathrm{HMB} / \mathrm{Creatine}$ Supplementation on Strength, Power, and Anthropometry of Highly Trained Athletes. The Journal of Strength and Conditioning Research, 21(2), 419. doi: 10.1519/R-15974.1

Pearson, D.R., Hamby, D.G., Russel, W., \& Harris, T. (1999). Long-term effects of creatine monohydrate on strength and power. Journal of Strength and Conditioning Research, 13(3), 187-192.

Pope, Z. K., Willardson, J. M., \& Schoenfeld, B. J. (2013). Exercise and blood flow restriction. Journal of Strength and Conditioning Research, 27(10), 2914-2926. doi: 10.1519/JSC.0b013e3182874721

Portal, S., Eliakim, A., Nemet, D., Halevy, O., \& Zadik, Z. (2010). Effect of HMB supplementation on body composition, fitness, hormonal profile and muscle damage indices. Journal of Pediatric Endocrinology \& Metabolism : JPEM, 23(7), 641-650. Retrieved from http://www.ncbi.nlm.nih.gov/pubmed/20857835

Rhea, M. R. (2004). Determining the Magnitude of Treatment Effects in Strength Training Research Through the Use of the Effect Size. The Journal of Strength and Conditioning Research, 18(4), 918. doi: $10.1519 / 14403.1$

Sanchez-Martinez, J., Santos-Lozano, A., Garcia-Hermoso, A., Sadarangani, K. P., \& Cristi-Montero, C. (2018). Effects of beta-hydroxy-beta-methylbutyrate supplementation on strength and body composition in trained and competitive athletes: A meta-analysis of randomized controlled trials. Journal of Science and Medicine in Sport, 21(7), 727-735. doi: 10.1016/j.jsams.2017.11.003

Slysz, J., Stultz, J., \& Burr, J. F. (2016). The efficacy of blood flow restricted exercise: A systematic review \&amp; meta-analysis. Journal of Science and Medicine in Sport, 19(8), 669-675. doi: 10.1016/j.jsams.2015.09.005

Souza-Junior, T. P., Willardson, J. M., Bloomer, R., Leite, R. D., Fleck, S. J., Oliveira, P. R., \& Simão, R. (2011). Strength and hypertrophy responses to constant and decreasing rest intervals in trained men using creatine supplementation. Journal of the International Society of Sports Nutrition, 8(1), 17. doi: 10.1186/15502783-8-17

Takarada, Y., Takazawa, H., Sato, Y., Takebayashi, S., Tanaka, Y., \& Ishii, N. (2000). Effects of resistance exercise combined with moderate vascular occlusion on muscular function in humans. Journal of Applied Physiology, 88(6), 2097-2106. doi: 10.1152/jappl.2000.88.6.2097

Wilson, J. M., Fitschen, P. J., Campbell, B., Wilson, G. J., Zanchi, N., Taylor, L., ... Antonio, J. (2013). International Society of Sports Nutrition Position Stand: beta-hydroxy-beta-methylbutyrate (HMB). Journal of the International Society of Sports Nutrition, 10(1), 6. doi: 10.1186/1550-2783-10-6

Wilson, J. M., Lowery, R. P., Joy, J. M., Andersen, J. C., Wilson, S. M. C., Stout, J. R., ... Rathmacher, J. (2014). The effects of 12 weeks of beta-hydroxy-beta-methylbutyrate free acid supplementation on muscle mass, strength, and power in resistance-trained individuals: a randomized, double-blind, placebo-controlled study. European Journal of Applied Physiology, 114(6), 1217-1227. doi: 10.1007/s00421-014-2854-5 